\title{
An Efficient Face Recognition System based on the Combination of Pose Invariant and Illumination Factors
}

\author{
S. Muruganantham \\ Assistant Professor, S.T.Hindu College, Nagercoil.
}

\author{
T. Jebarajan \\ Phd, Principal, Kings College of Engineering, \\ Chennai.
}

\begin{abstract}
In the preceding decade, Human face recognition has attracted significant consideration as one of the most effective applications of image analysis and understanding. Face recognition is one of the diverse techniques used for identifying an individual. Generally the image variations because of the change in face identity are less than the variations among the images of the same face under different illumination and viewing angle. Illumination and pose are the two major challenges, among the several factors that influence face recognition. Pose and illumination variations severely affect the performance of face recognition. Significantly less effort has been taken to tackle the problem of combined variations of pose and illumination in face recognition, though several algorithms have been proposed for face recognition from fixed points. In this paper we propose a face recognition method that is robust to pose and illumination variations. We first propose a simple pose estimation method based on 2D images, which uses a suitable classification rule and image representation to classify a pose of a face image. Then, the image can be assigned to a pose class by a classification rule in a lowdimensional subspace constructed by a feature extraction method. We propose a shadow compensation method that compensates for illumination variation in a face image so that the image can be recognized by a face recognition system designed for images under normal illumination condition. From the implementation result, it is evident that our proposed method based on the hybridization technique recognizes the face images effectively.
\end{abstract}

\section{Keywords}

Face recognition, Pose, illumination, Edge detection, Shadow compensation

\section{INTRODUCTION}

The rising importance of security and organization within various platforms currently available, identification and authentication methods turned out to key technology in different areas [1]. Recently, the use of biometrics has increased substantially in personal security and/or access control applications [3]. Biometrics is a technology, which is expected to replace traditional authentication methods that are easily stolen, forgotten and duplicated. Biometrics applied in fingerprints and iris scan deliver very reliable performance, yet human face stay as an attractive biometric due to its benefits over some of the other biometrics. Face recognition is non-intrusive and it needs no aid from the test subjects, while supplementary biometrics necessitates a subject's cooperation. For instance, in iris or fingerprint recognition, one should have a glance over an eye scanner or place their finger on a fingerprint reader [4].

In spite of very few biometric methods that holds the merits of both high accuracy and low intrusiveness, Face Recognition Technology has diverse potential over applications in the fields of information security, law enforcement and surveillance, smart cards, access control and more [5], [2], [6]. Face recognition seems to have aspiring challenges, since faces of different persons contribute to global shape characteristics, while face images of a single person is subject to substantial variations, which might engulf the measured inter-person differences. Such variation is due to the facts that integrate facial expressions, illumination conditions, pose, presence or absence of eyeglasses and facial hair, occlusion, and aging [7, 8]. The important significance of face recognition as a biometric is its throughput, convenience and non-invasiveness. Most of the face recognition investigation done till date uses $2 \mathrm{D}$ intensity images as the data format for processing. Success has been achieved at diverse levels over 2D face recognition research [9].

Face recognition scenarios have two major categories namely: Face verification is a one-to-one match that compares a query face image in counter with a gallery face image (The gallery face images are the images which have been stored in the database.) whose uniqueness being exposed. Face identification is one-tomany matching processes that weigh a query face image against all the gallery images in a face database to figure out the identity of the query face. The detection of the query image is rendered by spotting the image in the database that has the highest similarity with the query image [10]. Face recognition seems to be the major issue in abundant notorious regions of machine vision for about a decennium. Topical systems have progressed to be fairly accurate in recognition under constrained scenarios. On the other hand extrinsic imaging parameters such as pose, illumination, and facial expression still cause much complexity in correct recognition [11]. Generally, human face is similar in shape, have two eyes, a mouth and a nose. Each of these components makes different distinctive shadows and specularities depending on the direction of the lighting in a fixed pose. By using such characteristic, the lighting direction can be estimated and illumination variation can be compensated.

Moreover, in a practical application environment, the illumination variation is always coupled with other problem such as pose variation, which increases the complexity of the automatic face recognition problem. Changes in viewing conditions or rotations of faces bring difficulties to a face recognition system. There are two types of face rotations that must be considered; first one is the rotation of human face images in the image-plane and the second one is the rotation of faces out of the image plane (in-depth rotation). In the first case, 
face images can be easily normalized by detecting several landmarks in the face and then applying some basic transformations, while in the second case such normalization is impossible since some parts of faces may be occluded. Even for the same person, large difference of appearance is caused by pose variation. The difference is frequently more pronounced than that produced by different persons under the same pose [21]. Hence the degradation of conventional appearance-based methods for instance Eigen face etc., degrades severely when non frontal searches match against the registered frontal faces [20]. Amidst the several methods proposed to address the pose problem the extensively used method are view-based methods [19].

A number of illumination invariant face recognition approaches have been proposed in the past years. Existing approaches addressing the illumination variation problem fall into two main categories. The first category is "passive" approaches, since they attempt to overcome this problem by studying the visible spectrum images in which face appearance has been altered by illumination variations. The other category contains "active" approaches, in which the illumination variation problem is overcome by employing active imaging techniques to obtain face images captured in consistent illumination condition, or images of illumination invariant modalities. The changes induced by illumination are often larger than the differences between individuals, causing systems based directly on comparing images to misclassify input images. Approaches for handling variable illumination can be divided into four main categories: (1) extraction of illumination invariant features (2) transformation of images with variable illuminations to a canonical representation (3) modeling the illumination variations (4) utilization of some 3D face models whose facial shapes and albedos are obtained in advance [7].

Variable illumination is one of the most important problems in face recognition. The main reason is the fact that illumination, together with pose variation, is the most significant factor that alters the perception (appearance) of faces. Lighting conditions change largely between indoor and outdoor environments, but also within indoor environments. Thus, due to the 3D shape of human faces, a direct lighting source can produce strong shadows that accentuate or diminish certain facial features. Over past few decades, many face recognition methods have been developed. Often used feature extraction methods are principal component analysis (PCA) [12], [13] and linear discriminant analysis (LDA) [14], [15], [16], [17]. Another linear technique identified as Locality Preserving Projections (LPP) [18], [16], which finds an embedding that preserves local information, and gains a face subspace that best detects the essential face manifold structure.

Illumination and pose are the two main confronts to face recognition out of the diverse factors that affect face recognition. Pose and lighting variations considerably deteriorates the function of recognition. So to tackle this problem, the face is recognized under diverse lighting conditions by our proposed system. The rest of the thesis is structured as follows: section 2 describes some of the recent related works. Section 3 briefs the face recognition process based on a hybrid approach. Experimental results and analysis of the proposed methodology are discussed in Section 4. Finally, concluding remarks are provided in Section 5.

\section{LITERATURE REVIEW}

A handful of face recognition schemes, which employ template matching and model based techniques for improved performance, have been presented in the literature. Recently, incorporating pose invariant techniques into face recognition schemes to improve its performance and effectiveness has received a great deal of attention among researchers in face recognition community. A brief review of some recent researches is presented here.

One of the major challenges encountered by current face recognition techniques lies in the difficulties of handling varying poses, i.e., recognition off aces in arbitrary in-depth rotations. The face image differences caused by rotations are often larger than the inter-person differences used in distinguishing identities. Face recognition across pose, on the other hand, has great potentials in many applications dealing with un-cooperative subjects, in which the full power off ace recognition being a passive biometric technique can be implemented and utilized. Extensive efforts have been put into their search toward poseinvariant face recognition in recent years and many prominent approaches have been proposed by Xiao Zheng Zhang and Yong Sheng Gao [22]. Their paper provides a critical survey of researches on image-based face recognition across pose. The existing techniques were comprehensively reviewed and discussed. They were classified into different categories according to the methodologies in handling pose variations. Their strategies, advantages/disadvantages and performances were elaborated. By generalizing different tactics in handling pose variations and evaluating their performances, several promising directions for future research have been suggested.

Arashloo and Kittler [30] has addressed the problem of face recognition under arbitrary pose. A hierarchical MRF-based image matching method for finding pixel-wise correspondences between facial images viewed from different angles was proposed and used to densely register a pair of facial images. The goodness-of-match between two faces was then measured in terms of the normalized energy of the match which has a combination of both structural differences between faces as well as their texture distinctiveness. The method needs no training on non-frontal images and circumvents the need for geometrical normalization of facial images. It was also robust to moderate scale changes between images.

Dahm and Yongsheng Gao [31] have described that Many Face Recognition techniques focus on 2D-2D comparison or 3D-3D comparison; however few techniques explore the idea of crossdimensional comparison. Their paper presented a novel face recognition approach that implements cross-dimensional comparison to solve the issue of pose invariance. Their approach implements a Gabor representation during comparison to allow for variations in texture, illumination, expression and pose. Kernel scaling was used to reduce comparison time during the branching search, which determines the facial pose of input images. In their paper, they present a novel face recognition approach that utilizes 3D data to overcome changes in facial pose, while remaining non-intrusive. To achieve this, we use 3D textured head models in the gallery, as gallery data is generally taken from cooperative subjects (e.g. identification photos, mug shots). For the probes, we use 2D images, which can be taken 
from passive cameras such as ceiling mounted surveillance cameras. Together this gives us a cross-dimensional approach, combined with a non-intrusive nature.

Abhishek Sharma et al [32] has presented a simple but efficient novel H-eigenface (Hybrid-eigenface) method for pose invariant face recognition ranging from frontal to profile view. Heigenfaces were entirely new basis for face image representation under different poses and are used for virtual frontal view synthesis. The proposed method was based on the fact that face samples of same person under different poses are similar in terms of the combination pattern of facial features. H-eigenfaces exploit that fact and thus two H-eigenfaces under different poses capture same features of the face. Thereby providing a compact view-based subspace, which can be further used to generate virtual frontal view from inputted non-frontal face image using least square projection technique. The use of their proposed methodology on FERET and ORL face database shows an impressive improvement in recognition accuracy and a distinct reduction in online computation when compared to global linear regression method.

Choi Hyun Chul and Oh Se-Young [33] have proposed a realtime pose invariant face recognition algorithm from a gallery of frontal images only. First, they modified the second order minimization method for active appearance model (AAM). That allows the AAM to have the ability of correct convergence with little loss of frame rate. Second, they have proposed pose transforming matrix which can eliminate warping artifact of the warped face image from AAM fitting. That makes it possible to train a neural network as the face recognizer with one frontal face image of each person in the gallery set. Third, they propose a simple method for pose recognition by using neural networks to select proper pose transforming matrix.

Khaleghian and Rohban [34] has proposed an ensemble-based approach to boost performance of Tied Factor Analysis(TFA) to overcome some of the challenges in face recognition across large pose variations. They used Adaboost. $\mathrm{m} 1$ to boost TFA which has shown to possess state-of-the-art face recognition performance under large pose variations. They have employed boosting as a discriminative training in the TFA as a generative model. In their model, TFA was used as a base classifier for the boosting algorithm and a weighted likelihood model for TFA was proposed to adjust the importance of each training data. Moreover, a modified weighting and a diversity criterion are used to generate more diverse classifiers in the boosting process. Experimental results on the FERET data set demonstrated the improved performance of the Boosted Tied Factor Analysis (BTFA) in comparison with TFA for lower dimensions when a holistic approach was used.

\section{AN EFFICIENT FACE RECOGNITION SYSTEM}

Let $D$ be a database containing $N$ number of images, $\left\{I_{1}, I_{2}, \ldots, I_{N}\right\}$ and let $I$ be a database image of size $R \times S$. $D$, After inputting an image from the database $D$, the user must specify the type of the image i.e., whether it is pose invariant, illumination invariant or both pose and illumination variant. Depending on the image group precise by the user, any one of the following three processes is executed by the system.

\subsection{Face Detection}

In the recent years face detection have attained to a great extent. Various approaches were offered in excess of previous years in the field of face and eye detection. The position of the art techniques are emergence based methods, which include a lot of ways for object recognition such as neural networks (NN), support vector machines (SVM) etc. The beginning of face detection using neural network, by Rowley et al, in 1996, was an important work by that time [20]. Consequently, there are many other approaches. Interested reader may read the comprehensive survey paper by Yang, et al [21]. Viola and Jones [22] uses boosted cascade of simple Haar-like features introduced by Papageorgiou [24] and enhanced by Viola [22] and Lienhart [23] for object detection. This is one of the most discernible algorithms in face detection.

The Viola-Jones [22] face detection system makes use of a multi-scale multi-stage classifier that functions on image intensity information. This manner of face detection is operated in the existing system that doesn't require any previous calibration process. In general this loom scrolls a window across the image and consigns a binary classifier that discriminates between a face and the background. This classifier is regimented with a boosting machine learning meta-algorithm. It is endorsed as the fastest and most accurate pattern recognition method for faces in monocular grey-level images. They urbanized a real-time face detector comprising of a cascade of classifiers trained by AdaBoost. Every classifier workout with an integral image filter, which hark back of Haar Basis functions and can be processed very fast at any location and scale. This is important to speed up the detector. At each level in the cascade, a division of features is preferred using a feature choice procedure based on AdaBoost.

The process operates on professed primary images: each image element contains the sum of all pixels values to its upper left, allowing for constant-time outline of random rectangular areas [26].

Integral Image:-

For the original image $i(x, y)$, the primary image is defined as follow:

$$
i i(x, y)=\sum_{x^{\prime} \leq x} \sum_{y^{\prime} \leq y} i\left(x^{\prime}, y^{\prime}\right)
$$

Using the following pair recurrence:

$$
\begin{aligned}
& s(x, y)=s(x, y-1)+i(x, y) \\
& i i(x, y)=i i(x-1, y)+s(x, y)
\end{aligned}
$$

(Where $s(x, y)$ is the cumulative row sum, $s(x,-1)=0$ plus $i i(-1, y)=0$ ) the primary image can be processed in one pass over the original image. Using the primary image any rectangular sum can be computed in four array references (see Fig. 2). 


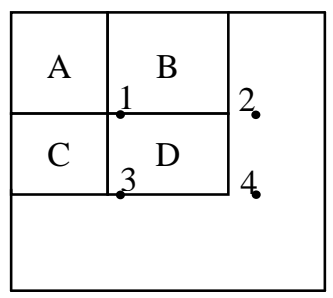

Figure 1. The sum of the pixels within rectangle D can be computed with four array references

The significance of the primary image at location 1 is the sum of the pixel in rectangle $A$. The value in location 2 is $A+B$, at location 3 is $A+C$, and at location 4 is $A+B+C+D$. The sum within $D$ can be computed as $4+1-(2+3)$.ViolaJones' modified AdaBoost algorithm is offered in pseudo code [25] as below.Viola-Jones' modified AdaBoost algorithm is offered in pseudo code [25] as below.

\section{The Modified Adaboost Algorithm}

- $\quad$ Given sample images $\left(x_{1}, y_{1}\right), \ldots,\left(x_{n}, y_{n}\right)$, where $y_{1}=0,1$ for negative and positive examples.

- Initialize weights $w_{1, i}=\frac{1}{2 m}, \frac{1}{2 l} \quad$ for $y_{1}=0,1$, where $m$ and $l$ are the numbers of positive and negative examples.

- For $t=1, \ldots, T$ :

1. Normalize the weights,

$$
w_{t, i} \leftarrow \frac{w_{t, i}}{\sum_{j=1}^{n} w_{t, j}}
$$

2. Choose the best weak classifier in regard to the weighted error

$$
\varepsilon_{t}=\min _{f, p, \theta} \sum_{i} w_{i} \mid h\left(x_{i}, f, p, \theta\right)-y_{i}
$$

3. Define $h_{t}(x)=h\left(x, f_{t}, p_{t}, \theta_{t}\right)$ where $f_{t}, p_{t}$ and $\theta_{t}$ are the minimizers of $\varepsilon_{i}$

4. Update the weights:

$$
w_{t+1, i}=w_{t, i} \beta^{1-e_{i}}
$$

Where $e_{i}=0$ if example $x_{i}$ is classified correctly and $e_{i}=1$ otherwise, and

$$
\beta_{t}=\frac{\varepsilon_{t}}{1-\varepsilon_{t}}
$$

- The final strong classifier is:

$$
\begin{aligned}
& C(x)=\left\{\begin{array}{l}
1 \quad \text { if } \sum_{t=1}^{T} \alpha_{t} h_{t}(x) \geq \frac{1}{2} \sum_{t=1}^{T} \alpha_{t} \\
0 \quad \text { otherwise }
\end{array}\right. \\
& \text { Where } \alpha_{t}=\log \frac{1}{\beta_{t}} \text {. }
\end{aligned}
$$

After detecting the face in the image all further analysis is placed in the upper region of the found face (ROI).

\subsection{Face Recognition}

There are three phases of proposed face recognition system namely 1) pose estimation, 2) shadow compensation, 3) face identification. For a face image with multiple variations, the pose of the face image is likely by using the projected pose estimation method. After assigning a face image to an appropriate pose class, the face image is processed by the outline compensation procedure modified for each pose class. These shadow compensated images are used for face identification by a classification rule. The projected method has the following advantages compared to other face recognition methods under clarification and pose variations. Unlike most of 2D image-based methods that contract with individual variation discretely, the proposed method handles both illumination and poses variations. Likewise, the proposed method, which is based on 2D images, does not require to estimate the face surface normals or the albedos, and thus there is no need for any special equipment such as a 3D laser scanner [35-37] or complicated calculation. The proposed shadow compensation method also does not include image deforming or iteration process. These make the proposed recognition system much simpler to implement, and this ease is an important factor for performing a face recognition system in real-time.

\subsubsection{Pose Estimation}

Within view-based face recognition [38, 39], the pose estimation is to categorize start point of reference into one of several distinct prientation classes, e.g., frontal, left/right profiles, etc. Along $i$ with the pose estimation methods based on 2D images such as the geometric methods, detector array methods, appearance template methods and subspace methods [40], we use the subspace method that schemes a check out image into a lowdimensional subspace to estimate its pose. We first divide the pose space into several pose classes from left profile to right profile. In view-based face recognition, the pose estimation stage is essential for face recognition performance since it is at the first stage in face recognition system to verify the pose class of an image. To make pose estimation more consistent against the variations in subjects and ecological alters, it is essential to find the characteristics that are mostly affected by pose variation. We use the geometrical distribution of facial components for pose estimation because the locations of facial components change depending on the pose. Through this information, we can estimate the pose and determine the pose class by a classification rule. In order to remove the redundant information for pose estimation, we transform a face image to an edge image. The block diagram for the whole projected method is offered in Fig. 2 . 


\section{Edge Detection}

In the allocation of facial components edge image is an effective representation. On the converse, in the edge image only the irregular shapes of facial components are present in the edge images, whereas the other information vanishes specifically the positions of facial components are improved in an edge image.raw images contain not only the allocation of facial components however the other information such as texture, graylevel intensity, and appearance variation of subjects, and these can act as noise in estimating pose. Several edge detection algorithms have been proposed in image processing area. Among them, we take on the Sobel edge detector which uses two convolution kernels, one to detect changes in vertical contrast and another to detect changes in horizontal contrast. The Sobel edge detector is effortless and the edges produced by the Sobel edge detector increase only the geometrical distribution of facial components eradicating needless edge shapes.

With pertain a discriminate characteristic mining method to these Sobel edge images from the images of training set, a subspace is raised for each of $K$ pose classes $\left\{P_{k} \mid k=1,2, \cdots, K\right\}$. The subspace for classification of the pose class is constructed by using a seperate feature extraction method. The pretense of each image projected into the subspace is classified by using the one nearest district rule with the $l_{2}$ norm as the distance metric, and a pose class $P_{k}, k=1,2, \cdots, K$ is assigned to each image.

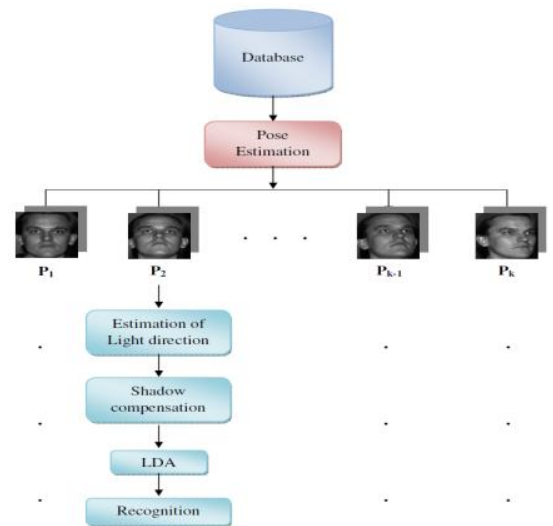

Fig.2: Block diagram of proposed face recognition method

\section{Shadow compensation}

On behalf of motion compensation, first we guess the direction of light for the images in each pose class. while the shape of a human face is more convex in azimuth than in elevation, we divide the directions of light into $L$ categories $\left\{C_{l} \mid l=1,2, \cdots, L\right\}$ from the left side to the right side. We denote the gray-level intensity of a face image of $H($ height $) \times W($ width $)$ pixels as $F I_{m, n}^{k, l}(x, y) \in \mathfrak{R}^{H \times W}$, where the subscripts $m=1,2, \cdots, M$ and $n=1,2, \cdots N_{(k, l)}$ denote the $n^{\text {th }}$ nth image of the $m^{\text {th }}$ individual when the direction of light belongs to category $C_{l}$ in the pose class $P_{k}$. Hence, the superscript $(k, l)$ denotes that the pose class of the image is $P_{k}$ and the direction of light belongs to $C_{l}$. Towards approximation of the direction of light, we make a binary image with a threshold $\frac{1}{H W} \sum_{x=1}^{H} \sum_{y=1}^{W} F I^{(k, l)}(x, y)$ which is the average value of the gray-level intensities, for each face image.

$\mathrm{C}_{4}$

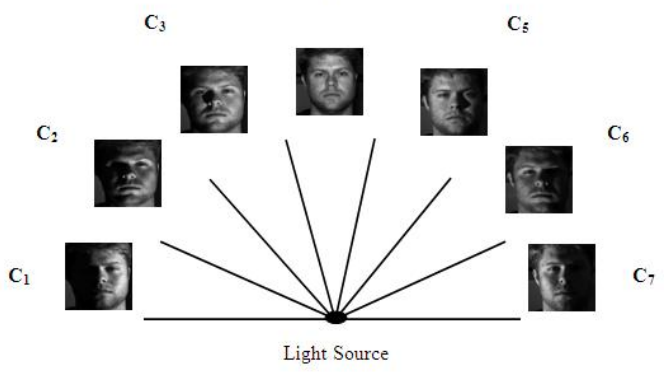

Fig. 3: Light direction categories

By means of these dual images, we consign a category value to the light category $C_{l}$. We evaluated this light track classification system with the Yale B database which provides information about the location of the flashlight for each image. In fig. 3 the light group changes from $\mathrm{C} 1$ to $\mathrm{C} 7$ as the light source moves from left

to

right. Diff ${ }_{m, n}^{k, l}(x, y)=F I_{m, n}^{k, r e f}(x, y)-F I_{m, n}^{k, l}(x, y)$ (7)

As the majority, human faces are comparable in shape, we can imagine that the gloom on facial images in the same pose class and the same illumination category are moreover similar in shape, and the difference image between the images with and without the shadows contains the information on the illumination condition. We select one of the images under the frontal illumination in each pose $P_{k}$ reference image $F I_{m, n}^{k, r e f}(x, y)$. The gray-level intensity $F I_{m, n}^{k, l}(x, y)$ at pixel $(x, y)$ varies depending on the light category, and is different from that of. $F I_{m, n}^{k, r e f}(x, y)$ We define the intensity difference between the images of $F I_{m, n}^{k, r e f}(x, y)$ and $F I_{m, n}^{k, l}(x, y)$ at each pixel $(x, y)$ as follows

$\operatorname{Diff}_{m, n}^{k, l}(x, y)=F I_{m, n}^{k, r e f}(x, y)-F I_{m, n}^{k, l}(x, y)$

The strength dissimilarity $\operatorname{Diff}_{m, n}^{k, l}$ of one person is inadequate to balance for the strength dissimilarities of another person's images under different illumination environment because $\operatorname{Diff}_{m, n}^{k, l}$ contains information about the illumination condition as well as unique features of each individual. So as to compensate for the intensity difference due to illumination variation, we need to eliminate the influence of features that are 
inherent to each individual. hence, we describe the average intensity difference $\operatorname{Diff}_{A}^{k, l}$ for the category $C_{l}$ in the pose class $P_{k}$ as follows:

$$
\begin{gathered}
\operatorname{Diff}_{A}^{k, l}=\frac{1}{M N(k, l)} \sum_{m=1}^{M} \sum_{n=1}^{N(k, l)} D_{m, n}^{(k, l)} \\
w_{l_{i}}=\frac{\operatorname{Dist}_{4-i}^{N}}{\sum_{i=1}^{3} \operatorname{Dist}_{i}^{N}}
\end{gathered}
$$

Note that there is no subscripts $m$ or $n$ in Diff ${ }_{A}^{k, l}$. Since this typical strength difference signifies the general attribute of the shadow in a face image for the direction of light belonging to category $C_{l}$, it can be applied to any face image in compensating for the shadow formed by the light belonging to the category $C_{l}$ in the pose class $P_{k}$. Since the direction of light can change continuously, it is moreover optimistic to expect that one average intensity difference contains enough information for shadow compensation in each pose class and light direction category. Once calculating the distances between the binary image of a test image and the binary images in each category $C_{l}$, the three nearest distances and their consequent groups are selected. The weight $w_{l_{i}}$, which entails the degree of involvement to the recompense, is determined based on these three nearest distances as the following.

$$
w_{l_{i}}=\frac{\operatorname{Dist}_{4-i}^{N}}{\sum_{i=1}^{3} \operatorname{Dist}_{i}^{N}}
$$

Then, we obtain the shadow remunerated image, $F I_{m, n}^{c(k, l)}$ of $F I \frac{c(k, l)}{m, n}$ with the typical differences as follows:

$$
F I_{m, n}^{c(k, l)}(x, y)=F I_{m, n}^{(k, l)}(x, y)+\sum_{i=1}^{3} w_{i} D_{A}^{k, l_{i}}(x, y)
$$

In general, the shadows can be remunerated by histogram equalization method. For the unprocessed image, the components of the histogram are concerted in the low side of the intensity scale. Even if the components with small strength values extend over a wide range by the histogram equalization, a huge portion still remains in the low side of the intensity scale. In the histogram of the shadow compensated image, pixels are quite evenly dispersed over the entire range of intensity values. It is known that an image, whose pixels be inclined not only to occupy the entire range of gray levels but also to be distributed uniformly, will have an appearance of high contrast and will reveal a large variety of gray tones. Hence, a face recognition system is expected to perform better with the shadow compensated images than the histogram steady images.

\section{RESULTS AND DISCUSSIONS}

Here in this segment, we have investigated the routine of our planned novel face recognition loom. Our planned loom is executed in Mat lab (7.10) and face recognition was achieved using the large set of Yale Database B under various pose and illumination conditions. The results show that our loom has an hopeful concert.

Database: The concert of illumination invariant face recognition is commonly assessed using the Yale face database B. Nine different poses 10 folks is present in the Yale face database B. 64 different illumination conditions exists for each pose. Single light source images of 10 subjects each seen fewer than 576 viewing conditions specifically, a total of 5850 images are present in the database. The steady results accomplished by the projected face recognition system are listed below.
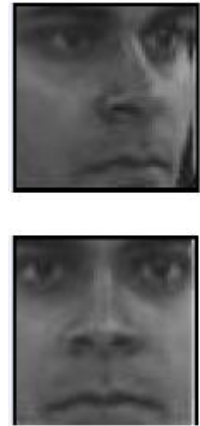

(b)

Fig. 4: Sample output obtained from the Posed invariant process a) non frontal face images b) Pose normalized images.
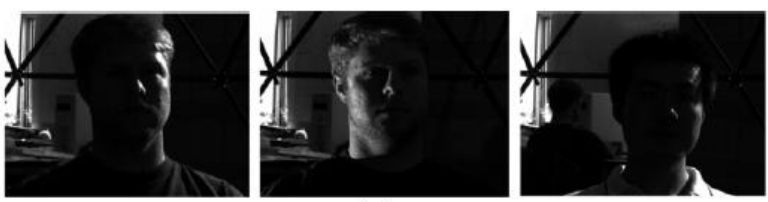

(a)
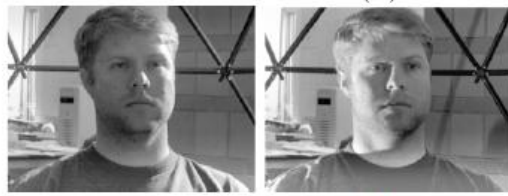

(b)

Fig. 5: Sample output obtained from the Illumination invariant process a) images with various illumination conditions $b$ ) Shadow remunerated images.

\section{Comparative analysis}

This associate segment nearby the proportional analysis of the projected approach. We evaluated the recognition accuracy of the proposed approach with some presented approaches. Computing the false acceptance rate (FAR) and false rejection rate (FRR) is the common way to measure the biometric recognition accuracy. FAR is the percentage of incorrect acceptances i.e., percentage of distance measures of different people's images that fall below the threshold. FRR is the percentage of incorrect rejections - i.e., percentage of distance measures of same people's images that exceed the threshold. The following equation is used to calculate the accuracy measurement of the overall approach, 


$$
\text { Accuracy }=100-\left[\frac{(F A R / F R R)}{2}\right]
$$

Genuine acceptance rate (GAR) is the general accuracy measurement of the approach. The following table gives the percentage of the recognition rates and the accuracy rates.

Table I: Comparison results of our proposed technique with

\begin{tabular}{|c|c|c|c|}
\hline \multicolumn{4}{|c|}{ Yale Database B } \\
\hline Methods & $\begin{array}{c}\text { FRR } \\
(\%)\end{array}$ & $\begin{array}{c}\text { FAR } \\
(\%)\end{array}$ & $\begin{array}{c}\text { Accuracy } \\
(\%)\end{array}$ \\
\hline $\begin{array}{c}\text { Face recognition based } \\
\text { on Pose invariant } \\
\text { condition }\end{array}$ & 6.82 & 8.25 & 93.4 \\
\hline $\begin{array}{c}\text { Face recognition based } \\
\text { on illumination invariant } \\
\text { condition }\end{array}$ & 5.84 & 7.51 & 94.2 \\
\hline Proposed Technique & 2.87 & 5.26 & 97.5 \\
\hline
\end{tabular}

some existing methods.

In this table, we study that our projected method has a lower value in both FAR and FRR error rate. Similarly, the proposed system has a higher accuracy compared the other two methods. This can be represented in the following graphs.

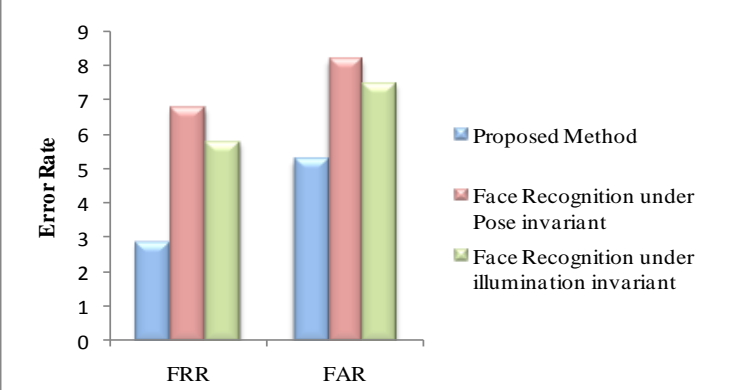

Fig.6: The comparative results of FAR and FRR on proposed technique and some existing techniques.

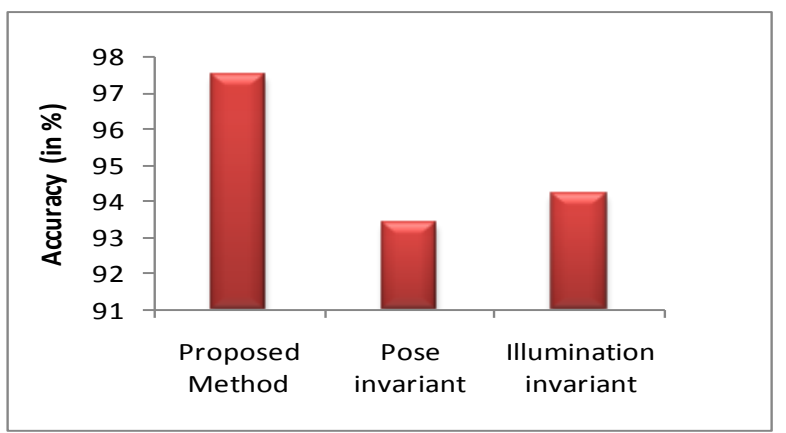

Fig. 7: The comparative results of accuracy on proposed system and some existing systems
Therefore it is evident that the proposed face recognition system resourcefully recognizes the face under various pose and illumination conditions.

\section{CONCLUSION}

In this paper, an efficient face recognition system based on pose and illumination invariant conditions was proposed. In this work, the face images were recognized by employing a hybridization of both the pose and illumination invariant condition. The efficiency of the proposed system was mainly due to the application of a hybrid process for face recognition. In hybrid process, we normalize both pose and illumination of the face image. This can be done by shadow compensation and pose normalization process. The implementation results showed that the face recognition process of the proposed method was more effective than existing methods that are either pose based or illumination based. The visualization graphs obtained for the recognition results illustrated this.

\section{REFERENCES}

[1] Alex Pentland and Tanzeem Choudhury, "Face Recognition for Smart Environments", IEEE Computer, 2000.

[2] Shang-Hung Lin, "An Introduction to Face Recognition Technology", Informing Science Special Issue On Multimedia Informing Technologies, Vol.3, No: 1, 2000.

[3] P. Phillips, "The FERET database and evaluation procedure for face recognition algorithms," Image and Vision Computing, Vol. 16, No. 5, pp.295-306, 1998.

[4] Sina Jahanbin, Hyohoon Choi, Rana Jahanbin, Alan C. Bovik, "Automated Facial Feature Detection and Face Recognition Using Gabor Features on Range and Portrait Images", ICIP08, pp: 2768-2771, 2008.

[5] Zhao, W. Chellappa, R., Phillips, P. J. and Rosenfeld, A., "Face Recognition: A Literature Survey", ACM Computing Survey, December, pp. 399-458, 2003.

[6] Gregory Shakhnarovich, Baback Moghaddam, "Face Recognition in Subspaces", .Springer, Heidelberg, May 2004

[7] Florent Perronnin, Jean-Luc Dugelay, Kenneth Rose, "A Probabilistic Model of Face Mapping with Local Transformations and Its Application to Person Recognition", IEEE Transactions on Pattern Analysis and Machine Intelligence, Vol. 27, No. 7, July 2005

[8] R. Chellappa, C. Wilson, and S. Sirohey, "Human and Machine Recognition of Faces: A Survey," Proc IEEE, vol. 83, No. 5, pp. 705-740, May 1995.

[9] Afzal Godil, Sandy Ressler and Patrick Grother, "Face Recognition using 3D Facial Shape and Color Map Information: Comparison and Combination", Biometric Technology for Human Identification, SPIE, vol. 5404, pp. 351-361, 2005

[10] Chao Li, Armando Barreto, "3D Face Recognition in Biometrics", Proceedings of the 2006 WSEAS International Conference on Mathematical Biology and Ecology (MABE '06), Miami, Florida, January 18-20, pp. 87 - 92, 2006. 
[11] Yuri Ivanov, Bernd Heisele, Thomas Serre, "Using Component Features for Face Recognition", AFGR04, pp.421-426, 2004.

[12] H. Moon, P.J. Phillips, "Computational and Performance aspects of PCA based Face Recognition Algorithms", Perception, vol. 30, pp. 303- 321, 2001.

[13] M. Turk and Pentland, "Face Recognition Using Eigenfaces", in Proc. IEEE International Conference on Computer Vision and Pattern Recognition, Maui, Hawaii, 1991.

[14] P. N. Belhumeur, J. P. Hespanha and D. J. Kriegman, "Eigenfaces vs. Fisherfaces: Recognition Using Class Specific Linear Projection", IEEE Trans. Pattern Analysis and Machine Intelligence, vol. 19, No. 7, pp. 711-720, 1997.

[15] J. Yang, Y. Yu, and W. Kunz, "An Efficient LDA Algorithm for Face Recognition", The sixth International Conference on Control, Automation, Robotics and Vision, Singapore, 2000.

[16] W. Zhao, R. Chellappa, and P. J. Phillips, "Subspace Linear Discriminant Analysis for Face Recognition", Technical Report CAR-TR-914, Center for Automation Research, University of Maryland, 1999.

[17] X. He and P. Niyogi, "Locality preserving projections, "In Advances in Neural Information Processing Systems. Cambridge, MA: MIT Press, 2003.

[18] Li, Ruidong; Zhu, Lei; Zou, Shaofang; "Face Recognition Based on an Alternative Formulation of Orthogonal LPP", Control and Automation, 2007, IEEE International Conference on May 30 2007-June 1, pp.2374 - 2376, 2007.

[19] A. Pentland, B. Moghaddam, and T. Starner, "View-based and modular eigenspace for face recognition", In Proc. of the IEEE Conf. Comput. Vision Pattern Recognition, pp. 84-91, 1994.

[20] X. Chai, S. Shan, X. Chen and W. Gao,"Locally Linear Regression for Pose-Invariant Face Recognition",IEEE Transactions On Image Processing, Vol. 16, No. 7, pp.17161725, July 2007.

[21] Z. Zhou, J. H. Fu, H. Zhang, and Z. Chen, "Neural network ensemble based view invariant face recognition", J. Comput. Study Develop., vol. 38, no. 9, pp. 1061-1065, 2001.

[22] Xiao zheng Zhang and Yong sheng Gao, "Face recognition across pose:A review", Pattern Recognition, Vol.42,pp. 2876--2896, 2009.

[23] S. R. Arashloo, and J. Kittler, "Hierarchical Image Matching for Pose-invariant Face recognition," In proc. of the International workshop on EMMCVPR '09, pp.56-69, 2009.

[24] Dahm, N. and Yongsheng Gao, "A Novel Pose Invariant Face Recognition Approach Using A 2D-3D Searching Strategy", In Proc. of the 20th International conference on Pattern Recognition (ICPR), pp.3967-3970, 2010.

[25] Abhishek Sharma, Anamika Dubey, A. N. Jagannatha and R. S. Anand, "Pose invariant face recognition based on hybrid global linear regression", Neural Computing \& Applications, Vol.19, No.8, pp.1227-1235, 2010.

[26] Choi Hyun Chul and Oh Se-Young, "Real-time PoseInvariant Face Recognition using the Efficient Second Order Minimization and the Pose Transforming Matrix", Advanced Robotics, Vol. 25, No.1-2, pp.153-174, 2011.

[27] Khaleghian, S. Rabiee and H.R. Rohban, M.H. ,"Face recognition across large pose variations via Boosted Tied Factor Analysis", In proc. of the IEEE workshop on Applications of Computer Vision (WACV), pp.190-195, 2011.

[28] Rowley, H.A., Baluja, S. and Kanade, T., "Neural NetworkBased Face Detection", IEEE Transactions on Pattern Analysis and Machine Intelligence, Vol. 20, No. 1, pp 2338, 1998.

[29] Yang, M-H., Kriegman, D., and Ahuja, N., "Detecting Faces in Images: A Survey", IEEE Transactions on Pattern Analysis and Machine Intelligence (PAMI), vol. 24, no. 1, pp. 34-58, 2002.

[30] Viola, P., Jones, M., "Rapid object detection using a boosted cascade of simple features", In Computer Vision and Pattern Recognition (CVPR 2001), 2001.

[31] R. Lienhart and J. Maydt, "An extended set of haar-like features for rapid object detection", In Proceedings. 2002 International Conference on Image Processing, vol. 1, pp. I900-I-903 2002.

[32] C.P. Papageorgiou, M. Oren, and T. Poggio, "A general framework for object detection", In Sixth International Conference on Computer Vision, pp. 555-562, 1998.

[33] Ole Helvig Jensen, "Implementing the Viola-Jones Face Detection Algorithm", Master's Thesis, Supervisor: Larsen, Rasmus, Technical University of Denmark, Department of Informatics and Mathematical Modeling, Image Analysis and Computer Graphics, Kongens Lyngby 2008.

[34] Qiong Wang, Jingyu Yang, and Wankou Yang, "Face Detection using Rectangle Features and SVM", International Journal of Intelligent Technology, Vol. 1, No. 3, pp. 228-232, 2006.

[35] Romdhani, S., Blanz, V., Vetter, T., "Face identification by fitting a 3D morphable model using linear shape and texture error functions", In Proc. of the European Conf. on Computer Vision - ECCV 2002, pp. 3-19, 2002.

[36] Romdhani, S., Blanz, V., Vetter, T., "Face recognition based on fitting a 3D morphable model", IEEE Trans. Pattern Anal. Machine Intell., Vol.25, No.9, pp.1-14, 2003.

[37] Romdhani, S., Vetter, T., "Efficient, robust and accurate fitting of a 3D morphable model", In: Proc. of the Internat. Conf. on Computer Vision - ICCV 2003, pp. 59-66, 2003.

[38] Li, Q., Ye, J., Kambhmettu, C., "Linear projection methods in face recognition under unconstrained illuminations: A comparative study", In Proc. Internat. Conf. on Computer Vision and Pattern Recognition - CVPR 2004, Vol. 2, pp. 474-481, 2004. 
[39] Pentland, A., Moghaddam, B., Starner, T., "View-based and modular eigenspaces for face recognition", In Proc. Internat. Conf. on Computer Vision and Pattern Recognition - CVPR 1994, pp. 84-91, 1994.

[40] Murphy-Chutorian, E., Trivedi, M., "Head pose estimation in computer vision: A survey", IEEE Trans. Pattern Anal. Machine Intell., Vol. 31, No.4, pp.609-626, 2009.

\section{AUTHORS PROFILE}

S.Muruganantham received the M.C.A degree in Manonmaniam Sundaranar University, Tamilnadu, India, in 1996. The M.Tech Degree in Manonmaniam Sundaranar University Tamilnadu, India, in 2004. In 1997 he joined the M.C.A Department, S.T.Hindu college, Tamilnadu, India as an Assistant Professor. His research interest includes Image processing, Operating System, Software Engineering. He has published international journals. He is currently a research scholar in the area of Face recognition. He is Life member of CSI, ACCS, IAENG, ISTE and IACSIT.

Dr. T. Jebarajan received the B.E Degree in Computer Science and Engineering in National Engineering College, Tamilnadu, India. The M.E. Degree in Computer Scinece and Engineering in Jadavpur University, Calcutta. The Ph.D in Computer science And Engineering from Manonmaniam Sundaranar University, Tamilnadu, India. He has worked as H.O.D. of Computer science and Engineering Department in Noorul Islam Deemed University, Kanyakumari District, Tamilnadu, India more than 15 years. Now he is working as a principal of KINGS College of Engineering, Chennai, Tamilnadu, India. He has National and International publication on the field of Computer Science. 